\title{
The Enrichment of BPMN Business Process Model with SBVR Business Vocabulary and Rules
}

\author{
Tomas Skersys, Lina Tutkute and Rimantas Butleris \\ Department of Information Systems, Kaunas University of Technology, Lithuania
}

\begin{abstract}
Aspects of business process models' integration with structured business vocabularies \& rules are analyzed in this paper. Despite the fact that business process (BP) modeling has its long-lasting tradition in various areas of application, this discipline remains in the constant process of improvement and issue-solving. The paper deals with one of such issues, namely, the existing gap between BP modeling and specification of business vocabularies $\&$ rules. If not dealt appropriately, this may lead to some issues while developing, reading and interpreting business models themselves, also to miscommunication issues within and among the organizations dependent on such models, and so on. The gap could be diminished by means of integration of BP models with business vocabularies \& rules; the paper presents some argumentation to back such statements. Later, basic principles of the approach for BPMN BP model integration with SBVR business vocabulary \& rules are presented and briefly described in this paper.
\end{abstract}

Keywords: business process, business process modeling, business vocabulary, business rule, SBVR, BPMN

\section{Introduction}

Business process (BP) modeling is recognized as one of the core disciplines in Business Process Management (BPM) life cycle. While some assume that BPM is as old as the existence of business itself, formalized process modeling techniques, such as DFD and IDEF, emerged only in the early 1970s. Next to BPM, Information Systems Development (ISD) is yet another area where BP modeling found its application, and the last decade's hype of various modeldriven automation solutions for ISD, especially Model-Driven Architecture-based [9], have finally imprinted BP modeling in ISD life cycle. Despite all the rich history of business process modeling, as well as its strong application perspectives, there are still some things to be improved in this discipline. Among these things, formalized support for Business Vocabularies (BV) and Business Rules (BR) are of particular interest and of the highest importance.

One of the newest standards among BP modeling languages is Business Process Model and Notation (BPMN) developed by OMG group [2]. It represents objects of the real world and seeks to eliminate the existing gap between modeled real world business processes and computerized processes. According to the researches carried out by different scientists and practitioners, BPMN models the dynamics of $\mathrm{BP}$ in a very proper way. However, BPMNbased BP modeling also lacks extensive integration with business vocabularies and rules. For this reason, BP modeling should be extended with new features for the identification (and, possibly, modeling) of such business entities as business terms, facts and business rules (BR). By definition, business rules define the semantics of business concepts, reactions to business events, constraints and preconditions on tasks and activities, as well as the rights and obligations of business actors. In other words, BR guide and constrain various aspects of business.

In J. C. Recker [13], the results from the interviews and some global survey confirmed that business people and IT professionals indeed have a great need to specify BR in their BP models. Nowadays, a common practice is to define BR separately, with a very loose (if at all) synchronization with BP models, goals and other 
formal models of the actual organization. Practice shows that BR are usually defined in a form of unstructured natural language and augment business models in a form of comments. It is obvious that such unstructured business knowledge cannot be passed to the next stages of BPM or communicated unambiguously within or outside the organization.

\section{Using BPMN and SBVR for the Integration of BP Models with Business Vocabularies \& Rules}

Due to the page number limitation, we will ommit basic introduction of BPMN and SBVR standards and will focus on the motivation of using these standards in our integration approach.

\subsection{Why BPMN?}

The primary goal of BPMN is to provide a modeling notation, which could be used and communicated by various people of different expertise: business analysts who model real world business processes, IT experts who are responsible for the implementation of the processes, and, finally, business people who will manage and monitor these processes or work according to the given instructions. The main diagram of BPMN is Business Process Diagram (BPD), which allows defining the process, its execution circumstances, some simple constraints, responsible actors and also to simulate the modeled process [2].

Compared to BPMN, software systems modelingoriented standards (like UML, IDEF) lack sufficient expressiveness. Moreover, such languages hold a number of concepts, constructions and rules that are excessive for BP modeling needs. Analysis of Bunge-Wand-Weber ontology [12] showed that, compared to other BP modeling languages, BPMN covers the largest amount of real world concepts and is well-understood and accepted by business experts $[4,10]$. The survey made by Wahl and Sindre [22] also indicated that BPMN is clearly understandable and wellsuited for BP modeling.

Also, BPMN fluently integrates into ModelDriven Architecture (MDA), which is a widely accepted standard by CASE tools developers.
Integration of BPMN into MDA is achieved via the meta-meta-model (Meta Object Facility - MOF), which is common with other OMG modeling standards, and is supported by OMG's constant efforts to integrate their standards on all levels of IS development. From MDA perspective, BPMN diagrams are a part of system's Computation Independent Model (CIM), also known as Business model.

\subsection{Why SBVR?}

The concept "Business rule" has quite a number of definitions. In this paper, a business rule (BR) is defined as a logical statement that defines or constrains some aspect of business in a concrete situation [21].

Business rules have a wide range of areas of application, such as BP management, IS development [16, 17], semantic technologies [19], artificial intelligence technologies, etc. Naturally, $\mathrm{BR}$ are expressed in a variety of forms and languages depending on the selected area - one can find rules buried straight into IS executable code or DB triggers [3], expressed in executable rule language of some BR management system (such as FICO Blaze Advisor or IMB Ilog) [18] or simply written down as natural language text. Neither of these forms is suitable for the use at the level of BP modeling, and this is exactly the place where BR should be identified and formally specified by business experts or system analysts. At this level, BR must be specified in a language, which is: well-structured and formal enough so that one could specify rules unambiguously and use these specified expressions in model transformations, rule exchange, execution and other activities alike; easily comprehendible and usable by business people who are the true owners of BR.

Practice shows that business people and even IS developers tend to express BR in unstructured natural language statements (so called business rumblings) followed by a loose list of common terms and definitions. During the last decade, people from Business Rules community such as R. G. Ross, B. von Halle and others worked on their own BR approaches [14, 21]; however, these efforts did not bring significant impact on the common practice of BR specification nor did they bridge the gap between BP modeling and BR specification. In 2008, OMG released 
"Semantics of Business Vocabulary and Business Rules" (SBVR) standard [15], which was welcomed by both business and IT sectors. The vision of SBVR is to express business knowledge in a controlled natural language, which is unambiguous and understandable to humans as well as to computer systems.

From the MDA perspective, business vocabularies and rules have to be defined at CIM level of MDA, i.e. in parallel with BP modeling. Indeed, both BP management and BR management focus on the improvement of organizational efficiency and effectiveness, but as technologies they have evolved separately [25]. Needless to say that formal "Business process model $\leftrightarrow$ Business vocabulary \& rules" $(\mathrm{BP} \leftrightarrow \mathrm{BV} / \mathrm{BR})$ integration methods, as well as their implementations are very welcome. SBVR is fully integrated into the OMG's Model-driven Architecture via Meta Object Facility (MOF) or Eclipse Meta-modeling Framework (EMF).

A few years ago, our research group finished a state-funded R\&D project VeTIS*. The main practical result of the project was VeTIS tool [11], which realized automated transformations of SBVR specifications written in SBVR Structured English into SBVR 1.0 XMI format and subsequently - into the UML Class Models with OCL constraints (in EMF UML 2.1.2 XMI). The project showed that it is possible to integrate SBVR with other OMG standards.

\subsection{State of the Art Research in the Area of $\mathrm{BP} \leftrightarrow \mathrm{BV} / \mathrm{BR}$ Integration}

Despite various discussions on how BR should be used in different areas of application, it is clear that BPM and BRM should not be treated as competing, but rather as complementary technologies. BR are now acknowledged as a critical component in BPM R\&D activities, due to the need to ensure the maximum flexibility and configurability of BP execution/monitoring solutions. Of course, modern standards-based $\mathrm{BP} \leftrightarrow \mathrm{BV} / \mathrm{BR}$ solutions could be utilized in many more areas of practical application (Figure 1).

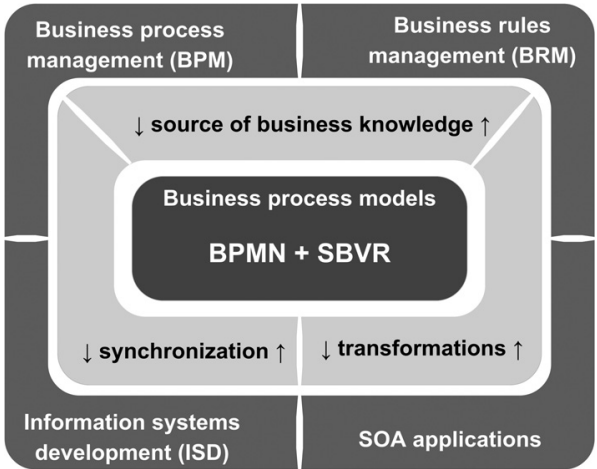

Figure 1. Basic areas of application for business process models (BPMN+SBVR).

In order to effectively integrate business vocabularies \& rules with BP models, the compatibility of BR and BP concepts must be assured. Early experiments to integrate BR and BP started a few decades ago. One of the first research works on this subject was some business rules methodology published by J. Krogstie et al. in 1991 [6]. Later, there were a number of various BP-BR integration-oriented research initiatives undertaken in various areas of application [5, 7]. However, the most relevant research works were undertaken after the advent of OMG's business modeling-oriented standards (BPMN and SBVR in particular).

J. Vanthienen and S. Goedertier proposed an approach to implement SBVR business rules into $\mathrm{BP}$ management life cycle using a serviceoriented architecture (SOA) $[7,20]$. They presented the architecture which consisted of three layers: BR and BR layer, services and components layer, application layer. S Ali et al. described BR as separate model used as an integral component of BP modeling [1]; here, BP were defined and arranged as directly dependent on BR model. M. Milanović et al. offered to integrate BPMN with R2ML [8]. They developed a new modeling language rBPMN (Rule-based Process Modeling Language); the idea behind rBPMN was to extend some existing elements of BPMN with the BR property. K. Zhao et al. analyzed semantic programing language (SPL) to facilitate the orchestration of semantic web services (SWS) [24]. The method integrated BR and BR using SWS.

The latest research made by $\mathrm{M}$. Muehlen et al. confirmed that BPMN has more accurate ad-

\footnotetext{
* Lithuanian State Science and Studies Foundation High Technology Development Program Project "Business Rule Solutions for Information Systems Development (VeTIS)". Download the tool at www.magicdraw.com/magicdraw_resources.
} 
equacy to the concepts of the real world processes, compared to Petri Nets, IDEF3 and EPC (Event-driven Process Chains) [10]. The background for this analysis was Bunge Wand Weber (BWW) ontology [23]. The same analytic principles were used to identify the overlapping of BR standards (SBVR, SRML) with Petri Net, IDEF3, EPC and BPMN. The results showed that combinations of BPMN with SRML and BPMN with SBVR provided the best representation power of business processes with minimum overlap [25]. Another fact mentioned in their investigations is a common organizational practice to write BR in a form of textual annotations in BP models. However, business people, as well as other interested parties, have to get used to model these constraints using appropriate BR standards to increase the effectiveness of $\mathrm{BR}$ in various application areas.

\section{Integration of BPMN BP Diagram with SBVR Business Vocabularies \& Rules}

\subsection{Mapping BPMN\&SBVR Meta-models}

Different strategies could be used to realize the integration of models, which are based on two different meta-models: one can merge together the existing meta-models into one base meta-model; one can reuse the existing metamodels enriching them with the required reference meta-elements; one can also incorporate some profiling techniques (UML's example) etc. However, in this development, we decided to leave the original meta-models as they are and develop supplementary mapping data structure, which would realize the link between those two meta-models (Figure 2). Any enrichment of the original model (e.g. BPMN BP diagram) with additional information (e.g. visualized business vocabulary entries and $\mathrm{BR}$ ) is realized via existing graphical and attributive elements by adding additional interpretation logic of those elements into the implementation of the solution itself. Such decision was based on the fact that even a slightest modification of widely-accepted meta-models, such as SBVR and BPMN, would significantly narrow the area of practical application of such solution. The VeTIS project, as well as other practical experimentations with the CASE tool
MagicDraw UML, proved the success of such integration approaches.

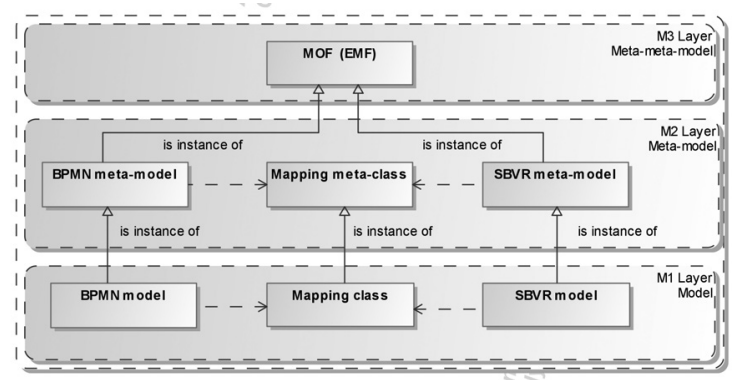

Figure 2. The principle of BPMN-SBVR integration in OMG's MDA.

In the suggested $\mathrm{BP} \leftrightarrow \mathrm{BV} / \mathrm{BR}$ integration approach, BPMN meta-model is linked with SBVR meta-model by mapping certain elements of one meta-model with particular elements of another meta-model via supplementary mapping data structure (Figure 2). Here, the Mapping class holds a set of attributes linking BPMN elements with the entries of SBVR specification: models' identifications, types and names of the linked elements.

Mapping sets of BPMN and SBVR elements on meta-models' level are presented in Table 1.

\begin{tabular}{|c|c|c|c|}
\hline BPMN Element & \begin{tabular}{|c|} 
SBVR \\
Noun Concept
\end{tabular} & \begin{tabular}{|c|} 
SBVR \\
Fact Type
\end{tabular} & $\begin{array}{l}\text { SBVR } \\
\text { Rule }\end{array}$ \\
\hline \multicolumn{4}{|l|}{ FlowObjects } \\
\hline Event & $\mathrm{x}$ & $\mathrm{x}$ & $\mathrm{x}$ \\
\hline Activity & $\mathrm{X}$ & $\mathrm{X}$ & $\mathrm{x}$ \\
\hline Gateway & $\mathrm{X}$ & $\mathrm{x}$ & $\mathrm{x}$ \\
\hline \multicolumn{4}{|c|}{ Connecting Obj. } \\
\hline Sequence Flow & $\mathrm{x}$ & $\mathrm{x}$ & $\mathrm{x}$ \\
\hline Message Flow & - & - & - \\
\hline Association & - & - & - \\
\hline Data Association & - & - & - \\
\hline \multicolumn{4}{|l|}{ Swimlanes } \\
\hline Lane & $\mathrm{x}$ & $\mathrm{x}$ & - \\
\hline Pool & $\mathrm{X}$ & $\mathrm{X}$ & - \\
\hline \multicolumn{4}{|l|}{ Artifacts } \\
\hline Data Object & $\mathrm{X}$ & $\mathrm{x}$ & $\mathrm{X}$ \\
\hline Data Store & $\mathrm{X}$ & $\mathrm{x}$ & - \\
\hline Group & $\mathrm{X}$ & - & - \\
\hline Text Annotation & - & - & - \\
\hline
\end{tabular}

Table 1. Mapping pairs of elements of SBVR and BPMN meta-models.

In the presented matrix, notion " $x$ " means that a certain element type from BPMN meta-model 
corresponds (or can be additionally specified) with some element type from SBVR meta-model. In other words, if the intersection of a certain BPMN element type $A$ (e.g. Activity) with some SBVR element type $B$ (e.g. Fact Type) is marked with " $x$ ", then a mapping pair " $A \leftrightarrow B$ " is embedded on a meta-models mapping level. For example, from the mapping matrix, one can state that some activity from a BP model (i.e. BPMN element type Activity) may have certain SBVR noun concepts, fact types and rules associated with that activity. In Table 1, cells marked with "--" indicate that there is no mapping between certain element types from SBVR and BPMN meta-models. A set of " $A \leftrightarrow B$ " mapping pairs can be viewed as a set of $\mathrm{BP} \leftrightarrow \mathrm{BV} / \mathrm{BR}$ mapping rules. These mappings are passed down to the modeling level where the instances of the Mapping class (Figure 2) are being created during the process BP modeling.

Let us assume that a "Supplier" performs some task named "Register Order" (Figure 5). Following our approach, two SBVR Business vocabulary (BV) concepts will be associated with the task "Register Order": a noun concept "order" and a fact type "supplier registers order"; in its turn, an actor (which is also the lane in BP diagram) "Supplier" will be associated with a noun concept "supplier" and also with a fact type "supplier registers order" from SBVR BV.

In case SBVR model already exists before the development of BP diagram (Figure 5), a business user will be interactively prompted by the system to reuse fragments of business knowledge from the existing SBVR specification, e.g.:

- Select a noun concept "customer" as a name for a certain lane in business process diagram (thus reusing the existing business concept);

- If the user specified data object "Order" on his own, then prompt for an automatic mapping of this data object with SBVR noun concept "order", which was already specified in $\mathrm{BV}$;

- When naming the task "Register Order", interactively prompt to use certain keywords (in this case, "Order");

- synonyms and synonymous forms of noun concepts and fact types would also be put into use; e.g. BPMN data object "Order", which was mapped with SBVR noun concept "order", will be mapped with "product_ order" and other synonyms of "order" specified in BV.

These and other intelligent features of "system $\leftrightarrow$ user" interaction are in the process of further development and implementation.

\subsection{Some Implementation Aspects of the BP $\leftrightarrow$ BV/BR Approach}

On technology level, the implementation of the proposed $\mathrm{BP} \leftrightarrow \mathrm{BV} / \mathrm{BR}$ approach may be viewed as an extension to our latest development - VeTIS tool [11], which may be used as a stand-alone tool or as a plug-in of the CASE tool MagicDraw UML. VeTIS tool's core features are:

1. Input and editing of SBVR Business vocabularies \& Rules with syntax validation;

2. Automatic transformation of SBVR models to UML class diagrams with OCL constraints.

At this point, our main objective is to extend the functionality of VeTIS tool by adding extensive $\mathrm{BP} \leftrightarrow \mathrm{BV} / \mathrm{BR}$ integration feature and thus allowing a user to work with SBVR Business vocabularies \& Rules in parallel with BP modeling and at the same time keep SBVR and BPMN models properly synchronized (Figure 3 ). MagicDraw UML fully supports BPMN-based BP modeling; therefore, SBVR and BPMN modeling activities are fluently integrated under the same working environment.

The approach uses standard MOF/EMF-based SBVR and BPMN meta-models supported by VeTIS and MagicDraw UML CASE tools accordingly. Mapping rules and interpretation logic are embedded into BPMN-SBVR synchronization plug-in (Figure 3 ). 


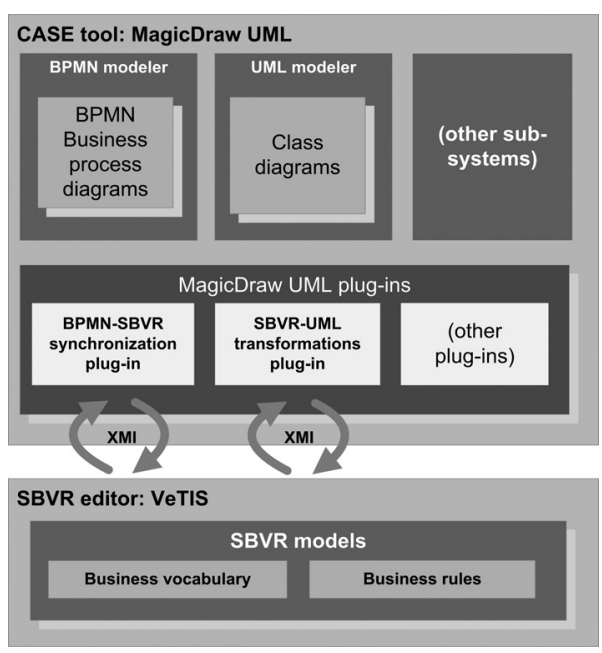

Figure 3. BPMN-SBVR integration using MagicDraw UML and VeTIS tools.

Linking of BPMN elements with SBVR specification is realized via common element Text Annotation attaching specific stereotype $<<$ $S B V R>>$ to it; $<<S B V R>>$ is a custom stereotype created for this approach (Figure 4).

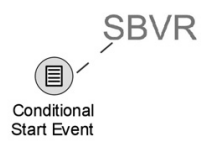

Figure 4. Stereotyped Text Annotation denoting the existence of SBVR expression associated with some BPMN element.
There may be more than one SBVR entry attached to one $<<S B V R>>$ text annotation. Double-clicking on such text annotation opens a list of SBVR entries associated with the particular BPMN element in VeTIS GUI window (Figure 5), from which SBVR entries may be viewed and edited.

Data exchange (import/export) between MagicDraw UML and VeTIS is realized via XMI format documents.

\subsection{The Algorithm of $\mathrm{BP} \leftrightarrow \mathrm{BV} / \mathrm{BR}$ Integration Approach}

In general, the proposed $\mathrm{BP} \leftrightarrow \mathrm{BV} / \mathrm{BR}$ approach may or may not be accompanied by a certain algorithm, which formally describes step-by-step the process of $\mathrm{BP} \leftrightarrow \mathrm{BV} / \mathrm{BR}$ integration. Due to page number limitation, we will not present this part of the approach in this paper. One can only mention that the algorithm is composed of three basic stages:

- Stage 1: Development and integration of BP diagram and Business vocabulary. The stage involves manual, semi-automated and fully-automated techniques for the systemassisted development of both, SBVR business vocabulary and BPMN BP diagram.

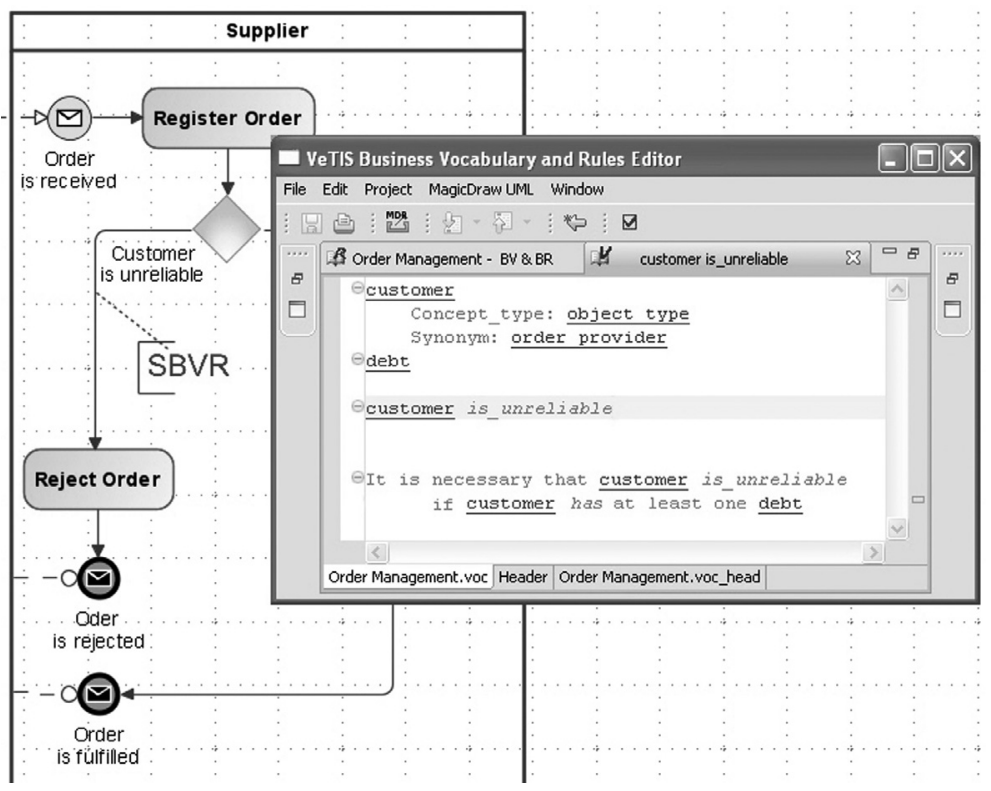

Figure 5. GUI window of VeTIS editor is called out by clicking on the stereotyped $<<S B V R>>$ text annotation in BPMN BP diagram (in MagicDraw UML CASE tool environment). 
- Stage 2: Augmentation of BP diagram with business rules. Unlike Stage 1, the augmentation of $\mathrm{BP}$ diagram with $\mathrm{BR}$ is quite straight-forward process where BR are linked to the elements of BP diagram via the stereotyped text annotations.

- Stage 3: Validation of the overall Business model with the domain expert.

\section{Conclusions}

Being a widely accepted BP modeling language, BPMN still lacks some important features that need to be implemented in order to reach the full potential of this standard. Authors of this paper emphasize the necessity to integrate BPMNbased BP modeling with business vocabularies and $\mathrm{BR}$, thus making a consistent BP model, which could be used in various areas of application, such as BP management (e.g. simulation), information systems development (especially, MDA-based ISD) etc.

Authors of this paper advocate the usage of SBVR standard to augment BPMN models with formally specified business vocabularies and business rules. Basic principles of such $\mathrm{BP} \leftrightarrow$ $\mathrm{BV} / \mathrm{BR}$ integration approach are presented in this paper. On the implementation level, the presented approach can be interpreted as an extension to some already developed VeTIS solution, which is composed of a full-featured SBVR editor and SBVR-to-UML transformation plug-in for the MagicDraw UML CASE tool.

When fully implemented, the proposed $\mathrm{BP} \leftrightarrow$ $\mathrm{BV} / \mathrm{BR}$ approach will enable a user to develop BPMN models synchronized with SBVR specifications, also enabling some level of the process automation, e.g. semi-automated development of SBVR Business vocabularies from the existing BPMN business process models, as well as some features of intelligent prompting to use parts of SBVR specifications while developing BPMN models.

\section{References}

[1] S. Ali, T. TORABI, S. BEn, Rule component specification for Business Process Deployment Database and Expert Systems Applications. In $18^{t} h$ International Workshop on Database and Expert Systems Applications, (2007) pp. 595-599. Germany.
[2] Business PRocess Model AND Notation (BPMN), v.2.0, OMG Document Number: formal/2011-01-03. http://www . omg.org

[3] L. Ceponiene, L. Nemuraite, G. Vedrickas, Separation of event and constraint rules in UML\&OCL models of service-oriented information systems. Information technology and control, vol. 38, no. 1 (2009), pp. 29-37.

[4] O. Glassey, A case study on process modelling - Three questions and three techniques. Decision Support Systems, vol. 44, no. 4 (2008), pp. 842-853.

[5] A. Kovacic, A. GrozniK, The Business Ruletransformation Approach. In Information Technology Interfaces, 1 (2004), pp. 113-117.

[6] J. Krogstie, P. McBrien, R. Owens, A. H. SELTVEIT, Information Systems Development Using a Combination of Process and Rule-based Approaches. In Third International Conference on Advanced Information Systems Engineering, SpringerVerlag, (1991) pp. 319-335. Trondheim, Norway.

[7] J. Meng, S. Y. W. Su, H. Lam, A. Helal, Achieving dynamic inter-organizational workflow management by integrating business processes, events and rules. In The $35^{\text {th }}$ Annual Hawaii Int. Conf. on System Sciences, HICSS, 2002.

[8] M. Milanovic, D. Gasevic, G. Wagner, Combining Rules and Activities for Modeling Service-based Business Processes. In $12^{\text {th }}$ Enterprise Distributed Object Computing Conf., (2008) pp. 11-22.

[9] Model-Driven ARChitecture (MDA) OMG, Version 2.0. October, 2003,

www. omg.org

[10] M. Muehlen, M. Indulska, G. Kamp, Business process and business rule modeling languages for compliance management: a representational analysis. In ACM Int. Conf., (334), (2007) pp. 127-132.

[11] L. Nemuraite, T. Skersys, A. Sukys, E. SINKEVIČIUS, L. ABLONSKIS, VETIS tool for editing and transforming SBVR business vocabularies and business rules into UML\&OCL models. In $16^{\text {th }}$ Int. Conf. on Information and Software Technologies, (2010) pp. 377-384. Lithuania.

[12] J. C. Recker, M. Indulska, M. Rosemann, P. GREEN, Do Process Modelling Techniques Get Better? A Comparative Ontological Analysis of BPMN. In $16^{\text {th }}$ Australasian Conference on Information Systems, (2005) Sydney, Australia.

[13] J. C. RECKER, Opportunities and constraints: the current struggle with BPMN. Business Process Management Journal, vol. 16, no. 1 (2010), pp. 181-201.

[14] R. Ross, Principles of the Business Rule Approach. ISBN 0-201-78893-4, Addison Wesley, 2003. 
[15] Semantics of Business Vocabulary and BusiNESS RULES (SBVR). OMG. v.1.0., 2008, http://www.omg.org

[16] T. SKERSYS, Business knowledge-based generation of the system class model. In Information technology and control, Kaunas, Technologija, ISSN 1392$124 X$, vol. 37, no. 2 (2008), pp. 145-153.

[17] S. SosunOVAS, O. VASILECAS, Transformation of business rules models in information systems development process. Scientific Papers. University of Latvia, Vol. 672, Database and Information Systems, ISSN 1407-2157, (2004) pp. 373-384.

[18] L. Tutkute, R. Butleris, Template-based business rules modelling from UML to executive code. Proc. of $8^{\text {th }}$ Int. Conf. on Perspectives in Business Informatics Research, (2009) pp. 7-14. Kristianstad.

[19] L. TutKute, R. Butleris, T. SKersys, An approach for the formation of leverage coefficientsbased recommendations in social network. In Information technology and control, Technologija, Kaunas, ISSN 1392-124X, 37(3) (2008), pp. 245254.

[20] J. VAnthienen, S. Goedertier, How Business Rules Define Business Processes. Business Rules Journal, vol. 8, no. 3 (2007).

[21] B. VON HALle, Business Rules Applied - Business Better Systems Using the Business Rules Approach. John Wiley \& Sons, Inc., ISBN 0-471-41293-7, 2002.

[22] T. WAHL, G. SindRE, An Analytical Evaluation of BPMN Using a Semiotic Quality Framework. In Proceedings of the CAiSE'05 Workshops, vol. 1, (2005) pp. 533-544. Universade do Porto, Porto.

[23] Y. WAND, R. WebER, An Ontological Model of an Information System. In IEEE Transactions on Software Engineering, vol. 16, (1990) pp. 1282-1292.

[24] K. ZhaO, S. YING, L. Zhang, L. Hu, Achieving Business Process and Business Rules Integration Using SPL Future Information Technology and Management Engineering (FITME). In Int. Conf., (2010) pp. 329-332.

[25] M. Zur Muehlen, M. IndulsKA, Modeling languages for Business Processes and Business Rules: A representational analysis. Information Systems Journal, vol. 35, no. 4 (2010), pp. 379-390.
Received: June, 2012 Accepted: August, 2012

Contact addresses: Tomas Skersys Department of Information Systems Kaunas University of Technology Studentu Str. 50-313a

Kaunas, Lithuania e-mail: tomas.skersys@ktu. It

Lina Tutkute

Department of Information Systems Kaunas University of Technology

Studentu Str. 50-313a Kaunas, Lithuania e-mail: lina.tutkute@ktu. lt

Rimantas Butleris Department of Information Systems Kaunas University of Technology Studentu Str. 50-313a Kaunas, Lithuania e-mail: rimantas. butleris@ktu.lt

TOMAS SKERSYS is an associate professor at the Department of Information Systems at Kaunas University of Technology, Lithuania. Research-wise his focus is on business process modeling and execution, business rules management and model-driven information systems development. His latest R\&D activities were related to OMG's standards UML, BPMN and SBVR. Tomas also reads lectures about model-driven information systems development and business process management.

LINA TUTKUTE is a $\mathrm{PhD}$ student at the Department of Information Systems at Kaunas University of Technology, Lithuania. Her current research is related to business process management, specification of business vocabularies \& rules, and also business rules management systems.

RIMANTAS BUTLERIS is a professor and head of the Department of Information Systems at Kaunas University of Technology. As a researcher and project coordinator, he is being constantly involved in several R\&D projects, international-and national-wide. Professor Butleris has high research and lecturing expertise in the areas of expert systems, requirements engineering, also in database and information systems development. He is co-author and author of over 130 scientific publications, including articles in international journals. He has been a general chair and program committee member on a number of international conferences held at Kaunas University of Technology and abroad. During the past 10 years, prof. Butleris was the coordinator or the chief manager of over 15 national or international research and development projects. 